Pacific Journal of Mathematics

SAMPLE FUNCTION REGULARITY FOR GAUSSIAN
PROCESSES WITH THE PARAMETER IN A HILBERT SPACE 


\section{SAMPLE FUNCTION REGULARITY FOR GAUSSIAN PROCESSES WITH THE PARAMETER IN A HILBERT SPACE}

\section{Peggy Tang Strait}

In this note, Gaussian processes $\left\{\xi_{t} ; t \in H\right\}$ where $H$ is the Hilbert space $l_{2}$ are considered. It is shown that if $T$ is a compact subset of a set of the form $\left\{\left(t_{1}, t_{2}, \cdots, t_{n}, \cdots\right)\right.$ : $\left.a_{n} \leqq t_{n} \leqq a_{n}+1 / 2^{n},\left(a_{1}, a_{2}, \cdots a_{n}, \cdots\right) \in H\right\}$ (thus including all compact subsets of $N$-dimensional Eulidean space), and there exists constants $\delta>0$ and $K>0$ such that

$$
E\left(\left|\xi_{t}-\xi_{s}\right|^{2}\right) \leqq \frac{K}{|\log \| t-s|||^{4+\delta}}
$$

for $t, s$ in $H$, then almost all sample functions of the process are continuous on $T$. Furthermore, if there are constants $\alpha>0$ and $K$ such that

$$
E\left(\left|\xi_{t}-\xi_{s}\right|^{2}\right) \leqq K\|t-s\|^{\infty}
$$

for all $t, s$ in $H$, then "almost all" sample functions of the process are Lipschitz- $\beta$ continuous on $T$ for $0<\beta<\alpha / 2$. The phrase "almost all" is used in the sense that the process defines a probability measure $\mu$ on the space $C_{T}$ of continuous or Lipschitz- $\beta$ continuous functions on $T$, such that for any $k$ points $t^{1}, t^{2}, \cdots t^{k}$ in $T$ and any Borel set $A$ in $k$-dimensional Euclidean space $R^{k}$

$$
\mu\left\{x \in C_{T}:\left(x\left(t^{1}\right), \cdots x\left(t^{k}\right)\right) \in A\right\}=P^{t^{1}, \cdots t^{k}}(A)
$$

where $P^{t^{1}, \cdots t^{k}}$ is the probability measure defined by the random vector $\left\{\xi_{t^{1}}, \cdots \xi_{t} k\right\}$. In the case where the process $\left\{\xi_{t}: t \in H\right\}$ is separable and is separated by the set of dyadic numbers in $H$, then the phrase "almost all" as defined here takes on the usual meaning.

In application, it is shown that the Brownian process in a Hilbert space defined by Paul Levy satisfies the latter condition for $\alpha=1$. Thus almost all sample functions are Lipschitz- $\beta$ continuous on $T$ for $0<\beta<1 / 2$ if $T$ is a compact set of the form described above. Furthermore, it is shown that Levy's result that almost all sample functions of this process are discontinuous in the Hilbert sphere may be extended to arbitrary noncompact subsets of the form $T=\left\{\left(t_{1}, t_{2}, \cdots\right.\right.$, $\left.\left.t_{n}, \cdots\right): a_{n} \leqq t_{n} \leqq b_{n}\right\}$.

We sufficient condition for Lipschitz- $\beta$ continuity of sample functions of Gaussian processes with the parameter in a Hilbert space. We use the following notations. $\left\{\xi_{t} ; t \in H\right\}$ denotes a real valued process where the parameter space $H$ is the Hilbert space $l_{2}$. $R^{N}$ is 
$N$-dimensional Euclidean space. $X$ is the space of real valued functions defined on $H$ or subsets $T$ of $H$, depending on the context. $C_{T}$ is the space of continuous (or Lipschitz- $\beta$ continuous) functions defined on T. $P$ is the complete probability measure on $X$ defined by the process $\left\{\xi_{t} ; t \in H\right\}$. For any $t^{1}, \cdots t^{k}$ in $H, p^{t^{1}, \cdots t^{k}}$ is the probability measure defined by the random vector $\left\{\xi_{t^{1}}, \cdots \xi_{t^{k}}\right\}$.

Lemma 1. Let $T$ be a compact subset of $H$ and $D$ a dense subset of T. If $\left\{\xi_{t} ; t \in H\right\}$ satisfies and

(1) $E\left(\left|\xi_{t}-\xi_{s}\right|^{\alpha}\right) \rightarrow 0$ as $\|t-s\| \rightarrow 0$ for some constant $\alpha>0$,

(2) $P\{x \in X: x$ is continuous (Lipschitz- $\beta$ continuous) in $D\}=1$, then there is a measure $\mu$ on the space $C_{T^{\prime}}$ such that

$$
\mu\left\{x \in C_{T}:\left(x\left(t^{1}\right), \cdots x\left(t^{k}\right)\right) \in A\right\}=P^{t^{1}, \cdots t^{k}}(A)
$$

for all finite sets $t^{1}, \cdots t^{k}$ in $T$ and Borel sets $A$ in $R^{k}$.

REMARK. If the process $\left\{\xi_{t}: t \in H\right\}$ is separable and is separated by $D$, then the conclusion of Lemma 1 is that $P\{x \in X: x$ is continuous in $T\}=1$, or in other words, almost all sample functions are continuous in the usual sense of the words "almost all".

Proof. Let $\bar{X}=\{x \in X: x$ is continuous (Lipschitz- $\beta$ continuous) in $D$ \}. Extend each $x$ in $\bar{X}$ by closure to a function $x^{*}$ which is continuous (Lipschitz- $\beta$ continuous) in $T$. Define map $\Pi: X \rightarrow C_{T}$ as follows:

$$
\Pi(x)= \begin{cases}x^{*} & \text { if } x \in \bar{X} \\ 0 & \text { if } x \notin \bar{X}\end{cases}
$$

We will show for fixed that $P\left\{x \in X: x^{*}(t)=x(t)\right\}=1$. This is clear if $t$ is in $D$. If $t$ is not in $D$, let $t^{1}, \cdots t^{j}, \cdots$ be a sequence in $D$ such that $t^{j} \rightarrow t$ as $j \rightarrow \infty$. Furthermore let $t^{1}, \cdots t^{j}, \cdots$ be chosen so that

$$
\sum_{j=1}^{\infty} P\left\{\left|x\left(t^{j}\right)-x(t)\right|>\varepsilon_{j}\right\} \leqq \sum_{j=1}^{\infty} \frac{E\left(\left|\xi_{t^{j}}-\xi_{t}\right|^{\alpha}\right)}{\varepsilon_{j}^{\alpha}}<\infty
$$

where $\varepsilon_{j} \rightarrow 0$ as $j \rightarrow \infty$.

Apply the Borel-Cantelli lemma to conclude that for almost all $x, x\left(t^{j}\right) \rightarrow x(t)$. Thus we have $x\left(t^{j}\right) \rightarrow x(t)$ for almost all $x, x^{*}\left(t^{j}\right)=x\left(t^{j}\right)$ for all $t^{j}$, and $x^{*}\left(t^{j}\right) \rightarrow x^{*}(t)$, for all $x$; hence $x^{*}(t)=x(t)$ for almost all $x$ in $X$, or $P\left\{x \in X: x^{*}(t)=x(t)\right\}=1$.

It follows that for any finite set $t^{1}, \cdots t^{k}, P\left\{x:\left(x\left(t^{1}\right), \cdots x\left(t^{k}\right)\right)^{-}=\right.$ $\left(x^{*}\left(t^{1}\right), \cdots x^{*}\left(t^{k}\right)\right\}=1$.

We will next show that $I$ is a measurable map of $X \rightarrow C_{r}$. Let 
$E$ be a Borel cylinder in $C_{T}$;

$$
E=\left\{x \in C_{T}:\left(x\left(t^{1}\right), \cdots x\left(t^{k}\right)\right) \in A\right\}
$$

where $A$ is a Borel set in $R^{k}$. If the set $A$ does not contain the point $(0,0, \cdots 0)$, then

$$
\begin{aligned}
\Pi^{-1}(E) & \\
= & \left\{x \in X:\left(x^{*}\left(t^{1}\right), \cdots x^{*}\left(t^{k}\right)\right) \in A\right\} \\
= & \left\{x \in X:\left(x^{*}\left(t^{1}\right), \cdots x^{*}\left(t^{k}\right)\right) \in A \text { and }\left(x^{*}\left(t^{1}\right), \cdots x^{*}\left(t^{k}\right)\right)=\left(x\left(t^{1}\right), \cdots x\left(t^{k}\right)\right)\right\} \\
& \cup\{" \prime \prime \prime \prime \\
= & \left\{x \in X:\left(x\left(t^{1}\right), \cdots x\left(t^{k}\right)\right) \in A\right\} \\
& -\left\{x \in X:\left(x\left(t^{1}\right), \cdots x\left(t^{k}\right)\right) \in A,\left(x^{*}\left(t^{1}\right), \cdots x^{*}\left(t^{k}\right) \neq\left(x(t) \cdots x\left(t^{k}\right)\right)\right\}\right. \\
& \cup\left\{x \in X:\left(x^{*}\left(t^{1}\right), \cdots x^{*}\left(t^{k}\right)\right) \in A \text { and }\left(x^{*}\left(t^{1}\right), \cdots x^{*}\left(t^{k}\right)\right) \neq\left(x\left(t^{1}\right), \cdots x\left(t^{k}\right)\right)\right\} .
\end{aligned}
$$

The first set on the right side of the last $=$ sign is a Borel cylinder in $X$ and is therefore measurable. The second and third sets have probability measure 0 and hence are also measurable sets. Thus $\Pi^{-1}(E)$ is a measurable set. On the other hand, if the set $A$ does contain the point $(0,0, \cdots 0)$, then

$$
\begin{aligned}
\Pi^{-1}(E) & =\left\{x \in X:\left(x^{*}\left(t^{1}\right), \cdots x^{*}\left(t^{k}\right)\right) \in A\right\} \\
& \cup\{x \in X: x \text { is discontinuous in } D\} .
\end{aligned}
$$

The probability of the second set on the right of this equality is zero, therefore again $\Pi^{-1}(E)$ is a measurable set. Consequently, $\Pi$ is a measurable map.

For Borel sets $E=\left\{x \in C_{T}:\left(x\left(t^{1}\right), \cdots x\left(t^{k}\right)\right) \in A\right\}$, define $\mu(E)=$ $P\left\{\Pi^{-1}(E)\right\}$. Thus

$$
\begin{aligned}
\mu(E) & =\mu\left\{x \in C_{T^{*}}:\left(x\left(t^{1}\right), \cdots x\left(t^{k}\right)\right) \in A\right\} \\
& =P\left\{x \in X:\left(x^{*}\left(t^{1}\right), \cdots x^{*}\left(t^{k}\right)\right) \in A\right\} \\
& =P\left\{x \in X:\left(x\left(t^{1}\right), \cdots x\left(t^{k}\right)\right) \in A\right\} \\
& =P^{t^{1}, \cdots t^{k}}(A) .
\end{aligned}
$$

Theorem 1. Let $\left\{\xi_{t} ; t \in H\right\}$ be a Gaussian process, $E\left(\xi_{t}\right)=0$, and $T$ a compact subset of the set $\left\{t: a_{n} \leqq t_{n} \leqq a_{n}+1 / 2^{n}\right\}$. If there are constants $\alpha>0$ and $k$ such that

$$
E\left(\left|\xi_{t}-\xi_{s}\right|^{2}\right) \leqq K\|t-s\|^{\alpha}
$$

for $t, s$ in $H$, then almost all sample functions of the process are Lipschitz- $\beta$ continuous in $T$ for $0<\beta<\alpha / 2$.

Proof. Since continuity in a compact set implies continuity in 
any compact subset of the set, we may assume without loss of generality that $T=\left\{t: 0 \leqq t_{m} \leqq 1 / 2^{m}, m=1,2, \cdots\right\}$. Let $D$ be the subset of all dyadic numbers in $T$, i.e., $t$ in $D$ implies that $t=$ $\left(t_{1}, t_{2}, \cdots, t_{m}, \cdots\right)$ where each $t_{m}$ is of the form $t_{m}=k / 2^{n}$. Write $h_{n}=$ $1 / 2^{n} ; \vec{k}=\left(k_{1}, k_{2}, \cdots, k_{m}, \cdots\right), \vec{j}=\left(j_{1}, j_{2}, \cdots, j_{m}, \cdots\right)$ where the $k_{m}$ and $j_{m}$ are positive integers. Write $\vec{k} h_{n}=\left(k_{1} h_{n}, k_{2} h_{n}, \cdots, k_{m} h_{n}, \cdots\right)$. Let $I_{n k}=\left\{t: k_{m} h_{n} \leqq t_{m} \leqq\left(k_{m}+1\right) h_{n}\right\}$. Observe that every dyadic number $t$ in $T \cap I_{n k}$ is of the form

$$
\begin{gathered}
k_{m} h_{n}+\sum_{r=1}^{M_{m}} \theta_{r} h_{n+r-1} \text { where } \theta_{r}=0 \text { or } 1 \text { for } m \leqq n \\
0+\sum_{r=1}^{M_{m}} \theta_{r} h_{n+r-1} \text { where } \theta_{r}=0 \text { or } 1 \text { for } m>n .
\end{gathered}
$$

Note that $t$ in $T$ implies that $0 \leqq t_{m} \leqq 1 / 2^{m}=h_{m}$. Use the notation $\varepsilon_{m}=(0,0, \cdots, \underset{\substack{\hat{\imath} \\ m \text { th place }}}{1,0,0}, \cdots)$.

Once we have chosen $\beta$ such that $0<\beta<\alpha / 2$, choose $\theta$ such that

$$
0<\beta<\theta<\alpha / 2 \text {. }
$$

Then for any $k, \varepsilon_{m}, h_{n}$ and

$$
\sigma_{n}^{2}=E\left(\left|\xi_{\left(\vec{k}+\varepsilon_{m}\right) h_{n}}-\xi_{\vec{k} h_{n}}\right|^{2}\right) \leqq k h_{n}^{\alpha}
$$

we have

$$
\begin{aligned}
& P\left\{\left|\xi_{\left(\vec{k}+\varepsilon_{m}\right) h_{n}}-\xi_{\vec{k} h_{n}}\right| \geqq\left\|\left(\vec{k}+\varepsilon_{m}\right) h_{n}-\vec{k} h_{n}\right\|^{\theta}\right\} \\
& =P\left\{\left|\xi_{\left(\vec{k}+\varepsilon_{m}\right) h_{n}}-\xi_{\vec{k} h_{n}}\right| \geqq h_{n}^{\theta}\right\}=2 \int_{h_{n}^{\theta}}^{\infty} \frac{1}{\sqrt{2 \pi \sigma_{n}^{2}}} \exp \left(-\frac{u^{2}}{2 \sigma_{n}^{2}}\right) d u \\
& =2 \int_{\frac{h_{n}^{\theta}}{\sigma_{n}}}^{\infty} \frac{1}{\sqrt{2 \pi}} \exp \left(-\frac{w^{2}}{2}\right) d w \quad \text { setting } w=\frac{u}{\sigma_{n}} \\
& \leqq 2 \int_{\frac{h_{n}^{\theta-(\alpha / 2)}}{\sqrt{\bar{k}}}}^{\infty} \frac{1}{\sqrt{2 \pi}} \exp \left(-\frac{w^{2}}{2}\right) d w \leqq \frac{2 \sqrt{k}}{\sqrt{2 \pi} h_{n}^{\theta-(\alpha / 2)}} \exp \left(-\frac{1}{2 k}\left(h_{n}^{\theta-(\alpha / 2)}\right)^{2}\right) .
\end{aligned}
$$

Let $\gamma=((\alpha / 2)-\theta)>0$, then we have

$$
\begin{aligned}
& P\left\{\left|\xi_{\left(\vec{k}+\varepsilon_{m}\right) h_{n}}-\xi_{\vec{k} n_{n}}\right| \geqq h_{n}^{\theta}\right\} \\
& \leqq \frac{2 \sqrt{k}}{\sqrt{2 \pi} h_{n}^{-\gamma}} \exp \left(-\frac{1}{2 k}\left(h_{n}^{-\gamma}\right)^{2}\right)=\frac{2 \sqrt{k}}{\sqrt{2 \pi} 2^{n \gamma} \exp \left(\frac{1}{2 k} 2^{2 n \gamma}\right)} .
\end{aligned}
$$

Hence,

$$
\sum_{n} P\left\{\max _{\vec{k}, m,\left(\vec{k}+\varepsilon_{m}\right) h_{n} \in D, \vec{k} h_{n} \in D}\left|\xi_{\left(\vec{k}+\varepsilon_{m}\right) h_{n}}-\xi_{\vec{k} h_{n}}\right| \geqq \frac{1}{2^{n \theta}}\right\}
$$




$$
\leqq \sum_{n}\left(2^{n(n-1) / 2}\right) n\left(\frac{2 \sqrt{k}}{\sqrt{2 \pi} 2^{n \gamma} \exp \left(\frac{1}{2 k} 2^{2 n \gamma}\right)}\right)<\infty .
$$

To prove this, simply observe that

$$
\left(2^{n(n-1) / 2} / \exp \frac{1}{2 k} 2^{2 n \gamma}\right)<1
$$

for $n$ sufficiently large, and $\sum_{n}\left(n / 2^{n \gamma}\right)<\infty$. Also, observe that the number $\left(2^{n(n-1) / 2}\right) \cdot n$ represents the maximum number of possible ways of choosing $\vec{k}$ and $m$ such that $\left(\vec{k}+\varepsilon_{m}\right) h_{n}$ and $\vec{k} h_{n}$ are in $D$; i.e., there are $n$ ways of choosing $m$ and for each of these there are $2^{n-1}$ ways of choosing $k_{1}, 2^{n-2}$ ways of choosing $k_{2}, \cdots 1$ way of choosing $k_{n}$, hence $n\left(2^{n-1} \cdot 2^{n-2} \cdots 2^{1}\right)=\left(2^{n(n-1) / 2}\right) \cdot n$.

Now we may apply the Borel-Cantelli lemma to conclude that for almost all sample functions $x$, there is an integer $N$ (dependent on $x$ ) such that $n \geqq N$ implies that

$$
\left|x\left(\left(\vec{k}+\varepsilon_{m}\right) h_{n}\right)-x\left(\vec{k} h_{n}\right)\right|<\frac{1}{2^{n \theta}}
$$

for all $\vec{k}, m$ such that $\left(\vec{k}+\varepsilon_{m}\right) h_{n}$ and $\vec{k} h_{n}$ are in $D$.

Let $x$ be a sample function for which (2) holds whenever $n \geqq N$. Let $t$ be in $I_{n k} \cap D, n \geqq N$. Then we may apply (1) and by repeated application of the triangle inequality

$$
\begin{aligned}
\mid x(t)- & x\left(\vec{k} h_{n}\right) \mid \\
\leqq & \sum_{r=1}^{M_{1}}\left(\frac{1}{2^{n+r-1}}\right)^{\theta}+\sum_{r=1}^{M_{2}}\left(\frac{1}{2^{n+r-1}}\right)^{\theta}+\cdots \sum_{r=1}^{M_{n}}\left(\frac{1}{2^{n+r-1}}\right)^{\theta} \\
& +\sum_{r=1}^{M_{n+1}}\left(\frac{1}{2^{n+r}}\right)^{\theta}+\sum_{r=1}^{M_{n}+2}\left(\frac{1}{2^{n+1+r}}\right)^{\theta}+\cdots \\
\leqq & n \sum_{r=1}^{\infty}\left(\frac{1}{2^{n+r-1}}\right)^{\theta}+\sum_{r=1}^{\infty} r\left(\frac{1}{2^{n+r}}\right)^{\theta} \leqq(n+1) \sum_{r=1}^{\infty} r\left(\frac{1}{2^{n+r-1}}\right)^{\theta} \\
= & \frac{n+1}{2^{n \theta}} \sum_{r=1}^{\infty} \frac{r}{2^{(r-1) \theta}}=\frac{n+1}{2^{n \theta}} M \text { where } M=\sum_{r=1}^{\infty} \frac{r}{2^{(r-1) \theta}}<\infty .
\end{aligned}
$$

Now observe that we have chosen $\theta$ such that $0<\beta<\theta<\alpha / 2$. This means that $\theta-\beta=\varepsilon>0$ so that

$$
\frac{n+1}{2^{n \theta}}=\frac{n+1}{2^{n \beta+n \varepsilon}} \leqq \frac{1}{2^{n \beta}} \text { for } n \text { sufficiently large . }
$$

Let $x$ be a sample function for which (2), (3), and (4) holds for $n \geqq N$. Let $s$ in $D$ be such that $\|s\| \leqq\left(1 / 2^{N}\right)$. We can find $n \geqq N$ such that $\left(1 / 2^{n}\right) \leqq\|s\| \leqq\left(1 / 2^{n-1}\right)$. Then for each $t$ in $D$ there are $\vec{k}$ and $\vec{j}$ such that 


$$
\begin{aligned}
& k_{m} h_{n} \leqq t_{m} \leqq\left(k_{m}+1\right) h_{n} \\
&\left(k_{m}+j_{m}\right) h_{n} \leqq t_{m}+s_{m} \leqq\left(k_{m}+j_{m}+1\right) h_{n}
\end{aligned}
$$

where $j_{m}=0$ or 1 for $m \leqq n$, and $j_{m}=0$ for $m>n, k_{m}=n$ for $m>n$. Hence it follows that

$$
\begin{aligned}
|x(t+s)-x(t)| & \leqq\left|x(t+s)-x\left((\vec{k}+\vec{j}) h_{n}\right)\right|+\left|x\left(\vec{k} h_{n}\right)-x(t)\right| \\
& \leqq \frac{(n+1) M}{2^{n \theta}}+\frac{n}{2^{n \theta}}+\frac{(n+1) M}{2^{n \theta}} \leqq \frac{3 M}{2^{n \beta}} \leqq \widetilde{A}\|s\|^{\beta}
\end{aligned}
$$

Hence we have shown that $\|s\| \leqq 1 / 2^{N}$ implies that $|x(t+s)-x(t)| \leqq$ $\tilde{A}\|s\|^{\beta}$ for all $t$ in $D$. Now, since $s$ in $D$ implies that $\|s\|^{2} \leqq$ $\sum_{m=1}^{\infty}\left(1 / 2^{m}\right)^{2}<\infty$ it is clear that there is a constant $A$ such that $|x(t+s)-x(t)| \leqq A\|s\|^{\beta}$ for all $t, s$ in $D$. Apply Lemma 1 to terminate the proof.

CoRollary 1. Let $\left\{\xi_{t} ; t \in R^{N}\right\}$ be a Gaussian process, $E\left(\xi_{t}\right)=0$, and $T$ a compact subset of $R^{N}$. If there are constants $\alpha>0$ and $K$ such that

$$
E\left(\left|\xi_{t}-\xi_{s}\right|^{2}\right) \leqq K\|t-s\|^{\alpha}
$$

for $t, s$ in $R^{N}$, then almost all sample functions of the process are Lipschitz- $\beta$ continuous in $T$ for $0<\beta<\alpha / 2$.

REMARK. Theorem 1 is an extension of a result of $\mathrm{Z}$. Ciesielski for the 1-dimensional case. [2]

2. A sufficient condition for continuity of sample functions of Gaussian processes with the parameter in a Hilbert space. The following lemma is a formulation of well known results [1] in analytic geometry and topology. It is used in the proof of Theorem 2.

Lemma 2. (a) Let a K-dimensional simplex be specified by the $K+1$ vertices $p_{0}, \cdots p_{k}$. Then every point $t$ in the simplex may be uniquely expressed as $t=\sum_{i=0}^{K} a_{i} p_{i}$ where $\sum_{i=0}^{K} a_{i}=1, a_{i} \geqq 0$.

(b) $A K$-dimensional parallelopiped can be divided into $K$ ! simplexes such that:

(1) they are disjoint except at the surfaces

(2) their union is the parallelopiped

(3) the vertices of the simplex are points that are vertices of the parallelopiped.

THEOREM 2. Let $\left\{\xi_{t} ; t \in H\right\}$ be a Gaussian process, $E\left(\xi_{t}\right)=0$, and $T$ a compact subset of the set $\left\{t: a_{n} \leqq t_{n} \leqq a_{n}+1 / 2^{n}\right\}$. If there are 
constants $\delta>0$ and $K$ such that

$$
E\left(\left|\xi_{t}-\xi_{s}\right|^{2}\right) \leqq \frac{K}{|\log \|t-s\||^{4+\delta}}
$$

for $t, s$ in $H$, then almost all sample functions of the process are continuous in $T$.

Proof. As in Theorem 1, assume that $T=\left\{\left(t_{1}, \cdots t_{n}, \cdots\right): 0 \leqq\right.$ $\left.t_{n} \leqq 1 / 2^{n}\right\}$. Let $T_{n}=\left\{\left(t_{1}, t_{2}, \cdots t_{n}\right)\right.$ : there is $t=\left(t_{1}, \cdots t_{n}, t_{n+1}, \cdots\right)$ in $T\}$. For each $n$, let $G_{n}=\left\{\left(a_{1}, a_{2}, \cdots a_{n}\right):\left(a_{1}, \cdots a_{n}\right)\right.$ in $T_{n}$ and $a_{i}=$ $\left.j / 2^{n}, j=0,1,2, \cdots 2^{n}\right\}$. By Lemma $2, G_{n}$ consists of the vertices of less than $2^{n n} n$ ! simplexes in $n$-dimensional space.

For each integer $n$, and sample function $x(t)$ we shall define a continuous function $\left(\Pi_{n}(x)\right)(t)$ as follows: Consider an arbitrary but fixed point $t=\left(t_{1}, t_{2}, \cdots\right)$ in $T$. Write $\left(t_{1}, \cdots t_{n}\right)=p^{(n)}$. (We will also let $p^{(n)}=\left(t_{1}, \cdots t_{n}, 0,0, \cdots\right)$ whenever appropriate.) Let $n$ be fixed. Then either $p^{(n)}$ is on the surface of a simplex defined by points in $G_{n}$, or else it is interior to a simplex with vertices say $\left(p_{0}\right.$, $p_{1}, \cdots p_{n}$ ) where each $p_{i}$ is in $G_{n}$. In either case, $p^{(n)}$ may be uniquely expressed as

$$
p^{(n)}=\sum_{i=1}^{n} a_{i} p_{i} \text { where } \sum_{i=0}^{n} a_{i}=1 ; a_{i} \geqq 0 .
$$

For each sample function $x(\cdot)$, we may define a function $\Pi_{n}(x)$ as follows.

$$
\left(\Pi_{n}(x)\right)(t)=\sum_{i=0}^{n} a_{i} x\left(p_{i}\right)
$$

where the points $p_{i}$ and the coefficients $a_{i}$ are chosen as described above. $\Pi_{n}(x)$ is clearly a continuous function of $t$ for $t$ in $T$.

We next show that for almost all $x,\left\{\Pi_{n}(x)\right\}$ forms a Cauchy sequence in the complete space of continuous functions $f$ on $T$ with norm defined by $\|f\|=\max _{t \in T}|f(t)|$.

In estimating $\left\|\Pi_{n}(x)-\Pi_{n-1}(x)\right\|$, for a fixed $t=\left(t_{1}, t_{2}, \cdots t_{n}, t_{n+1}\right.$, $\left.t_{n+2}, \cdots\right)$ in $T$, write $p=\left(t_{1}, \cdots t_{n-1}\right) \stackrel{\text { or }}{=}\left(t_{1}, \cdots t_{n-1}, 0,0, \cdots\right)$ and $q=$ $\left(t_{1}, \cdots t_{n-1}, t_{n}\right) \stackrel{\text { or }}{=}\left(t_{1}, \cdots t_{n-1}, t_{n}, 0,0, \cdots\right)$. Then according to the above discussion, we can express $p$ and $q$ as $p=\sum_{i=0}^{n-1} a_{i} p_{i} ; q=\sum_{i=0}^{n} b_{i} q_{i}$ where $\sum_{i=0}^{n-1} a_{i}=1, \sum_{i=0}^{n} b_{i}=1, a_{i} \geqq 0, b_{i} \geqq 0$, and where $\left(p_{0}, \cdots p_{n-1}\right)$ and $\left(q_{0}, \cdots q_{n}\right)$ are the vertices of the simplexes determined by $p$ and $q$ respectively in $G_{n-1}$ and $G_{n}$.

$$
\left(\Pi_{n}(x)\right)(t)=\sum_{i=1}^{n} b_{i} x\left(q_{i}\right) ;\left(\Pi_{n-1}(x)\right)(t)=\sum_{i=0}^{n-1} a_{i} x\left(p_{i}\right)
$$




$$
\begin{aligned}
\left|\left(\Pi_{n}(x)\right)(t)-\left(\Pi_{n-1}(x)\right)(t)\right| & =\left|\sum_{i=0}^{n} b_{i} x\left(q_{i}\right)-\sum_{i=0}^{n-1} a_{j} x\left(p_{j}\right)\right| \\
& \leqq \max _{i, j}\left|x\left(q_{i}\right)-x\left(p_{j}\right)\right|
\end{aligned}
$$

This last inequality is proved as follows:

$$
\begin{aligned}
\left|\sum_{i=0}^{n} b_{i} x\left(q_{i}\right)-\sum_{j=0}^{n-1} a_{j} x\left(p_{j}\right)\right| & =\left|\sum_{i} b_{i}\left(x\left(q_{i}\right)-\sum_{j} a_{j} x\left(p_{j}\right)\right)\right| \\
& \leqq \max _{i}\left|x\left(q_{i}\right)-\sum_{j} a_{j} x\left(p_{j}\right)\right| \\
& =\max _{i}\left|\sum_{j} a_{j}\left(x\left(q_{i}\right)-x\left(p_{j}\right)\right)\right| \\
& \leqq \max _{i} \max _{j} \mid x\left(q_{i}\right)-x\left(p_{j}\right) \\
& =\max _{i, j}\left|x\left(q_{i}\right)-x\left(p_{j}\right)\right| .
\end{aligned}
$$

Observe also that because of the way the $p_{j}$ and $q_{i}$ are chosen, $\left|q_{i}-p_{j}\right| \leqq\left(\sqrt{n} / 2^{n-1}\right)$.

Hence,

$$
\begin{aligned}
\left\|\Pi_{n}(x)-\Pi_{n-1}(x)\right\| & =\max _{t \in T}\left|\left(\Pi_{n}(x)\right)(t)-\left(\Pi_{n-1}(x)\right)(t)\right| \\
& \leqq \max _{s}\left\{\max _{i, j}\left|x\left(q_{i}\right)-x\left(p_{j}\right)\right|\right\}
\end{aligned}
$$

where $S$ is the following set. Let $\left(p_{0}, \cdots p_{n-1}\right)$ denote a simplex in $G_{n-1}$ with vertices $p_{0}, \cdots p_{n-1}$. Let $\left(q_{0}, \cdots q_{n-1}, q_{n}\right)$ denote a simplex in $G_{n}$ with vertices $q_{0}, \cdots q_{n-1}, q_{n}$. Then $S$ is the set of all pairs of simplexes $\left(p_{0}, \cdots p_{n-1}\right)$ and $\left(q_{0}, \cdots q_{n-1}, q_{n}\right)$ such that

$$
\left|q_{i}-p_{j}\right| \leqq \frac{\sqrt{n}}{2^{n-1}}, i=1, \cdots n, j=1, \cdots n-1 .
$$

Since the process is Gaussian, we have

$$
\begin{aligned}
& P\left\{x:\left|x\left(q_{i}\right)-x\left(p_{j}\right)\right|>a_{n}\right\} \\
& \quad=2 \int_{a_{n}}^{\infty} \frac{1}{\sqrt{2 \pi} \sigma_{n}} \exp \left(-\frac{u^{2}}{2 \sigma_{n}^{2}}\right) d u=2 \int_{\frac{a_{n}}{\sigma_{n}}}^{\infty} \frac{1}{\sqrt{2 \pi}} \exp \left(-\frac{w^{2}}{2}\right) d w
\end{aligned}
$$

where

$$
\begin{aligned}
\sigma_{n}^{2}=E\left(\left|\xi_{q_{i}}-\xi_{p_{j}}\right|^{2}\right) & \leqq \frac{K}{|\log | q_{i}-p_{j} \|^{4+o}} \\
& \leqq \frac{2}{\sqrt{2 \pi} \frac{a_{n}}{\sigma_{n}}} \exp \left(-\frac{1}{2}\left(\frac{a_{n}}{\sigma_{n}}\right)^{2}\right)
\end{aligned}
$$

\section{Hence}




$$
P\left\{x:\left\|\Pi_{n}(x)-\Pi_{n-1}(x)\right\|>a_{n}\right\} \leqq \frac{\left(2^{n \cdot n} n !\right)(n+1)^{2} 2}{\sqrt{2 \pi} \frac{a_{n}}{\sigma_{n}} \exp \left(\frac{1}{2}\left(\frac{a_{n}}{\sigma_{n}}\right)^{2}\right)}
$$

Let

$$
a_{n}=\frac{\sqrt{K} n^{1+\varepsilon}}{(n-1)^{2+\delta / 2}}\left(\left|\log 2-\frac{\log \sqrt{n}}{\mathrm{n}-1}\right|\right)^{2+\delta / 2} ; \frac{a_{n}}{\sigma_{n}} \geqq n^{1+\varepsilon}
$$

where $0<\varepsilon<\delta / 2$ so that $\sum a_{n}<\infty$. Since $\left|q_{i}-p_{j}\right| \leqq\left(\sqrt{n} / 2^{n-1}\right)$,

$$
\begin{aligned}
\sigma_{n} & \leqq \frac{\sqrt{K}}{|\log | q_{i}-p_{j}||^{4+\delta}} \leqq \frac{\sqrt{\bar{K}}}{\left|\log \sqrt{n} / 2^{n-1}\right|^{4+\delta}} \\
& =\frac{\sqrt{K}}{(n-1)^{2+\delta / 2}\left|\frac{\log \sqrt{n}}{n-1}-\log 2\right|^{2+\delta / 2}} .
\end{aligned}
$$

Thus

$$
\begin{aligned}
\sum_{n} P\{x & \left.:\left\|\Pi_{n}(x)-\Pi_{n-1}(x)\right\|>a_{n}\right\} \\
& \leqq \sum_{n} \frac{\left(2^{n \cdot n} n !\right)(n+1)^{2} 2}{\sqrt{2 \pi} \frac{a_{n}}{\sigma_{n}} \exp \left(\frac{1}{2}\left(\frac{a_{n}}{\sigma_{n}}\right)^{2}\right)} \\
& \leqq \sum_{n} \frac{\left(2^{n \cdot n} n !\right)(n+1)^{2} 2}{\sqrt{2 \pi} n^{1+\varepsilon} \exp \left(\frac{1}{2}\left(n^{1+\varepsilon}\right)^{2}\right)} \\
& =\sum_{n} \frac{2^{n \cdot n} n !(n+1)^{2} 2}{\sqrt{2 \pi} n^{1+\varepsilon} \exp \left(\frac{1}{2} n(2+2 \varepsilon)\right)} \\
& <\sum_{n} \frac{2^{n \cdot n} n^{n}(n+1)^{2} \cdot 2}{\sqrt{2 \pi} n^{1+\varepsilon} \exp \left(\frac{1}{2} n(2+2 \varepsilon)\right)} .
\end{aligned}
$$

Observe that

$$
\frac{2^{n \cdot n} n^{n}(n+1)^{2}}{\exp \left(\frac{1}{2} n(2+2 \varepsilon)\right)}<\frac{2^{n \cdot n} \cdot 2^{n \cdot n} \cdot 2^{n \cdot n}}{\exp \left(\frac{1}{2} n^{2} n^{2 \varepsilon}\right)}<1
$$

for $n$ sufficiently large. Hence

$$
\begin{aligned}
\sum_{n} P\{x & \left.:\left\|\Pi_{n}(x)-\Pi_{n-1}(x)\right\|>a_{n}\right\} \\
& <\sum_{n} \frac{2^{n \cdot n} n^{n}(n+1)^{2} \cdot 2}{\sqrt{2 \pi} n^{1+\varepsilon} e^{n^{2+2 \varepsilon}}}<M+\sum_{n=N}^{\infty} \frac{e}{n^{1+\varepsilon}}<\infty .
\end{aligned}
$$

Hence, by the Borel-Cantelli lemma, for almost all sample func- 
tions $x$,

$$
\left\|\Pi_{n}(x)-\Pi_{n-1}(x)\right\| \leqq a_{n}
$$

for $n$ sufficiently large ( $n$ dependent on $x$ ). Thus,

$$
\begin{aligned}
\left\|\Pi_{n+r}-\Pi_{n}(x)\right\| & \leqq \sum_{j+1}^{r}\left\|\Pi_{n+j}(x)-\Pi_{n+j-1}(x)\right\| \\
& \leqq \sum_{j=1}^{r} a_{n+j} \rightarrow 0 \text { as } n \rightarrow \infty .
\end{aligned}
$$

$\left\{\Pi_{n}(x)\right\}$ is therefore a Cauchy sequence for almost all $x$, and hence converges to a continuous function on $T$ for almost all $x$.

Let $D$ be the collection of elements of $T$ of the form $t=\left(t_{1}, t_{2}, \cdots\right.$ $\left.t_{n}, 0,0, \cdots\right)$ where $\left(t_{1}, \cdots t_{n}\right)$ is in $G_{n}$ for some $n$. Clearly $D$ is dense in $T$. The limit function continuous on $T$ for almost every $x$ coincides with $x$ on $D$. Thus almost all $x$ are continuous in $D$ and Lemma 1 applies.

REMARK. Theorem 2 is an extension of Kolmogorov's well known theorem to the Hilbert space. [7]

3. Extension of results to general processes with the parameter in a Hilbert space.

THEOREM 3. Let $\left\{\xi_{t} ; t \in H\right\}$ be a stochastic process with parameter space $H$. If there are constants $\delta>0, K>0$ such that for each positive integer $n$ there is a $Q_{n}$ such that $(1 / 2) \delta Q_{n-1}>\left(Q_{n}-Q_{n-1}\right)$ and $E\left(\left|\xi_{t}-\xi_{s}\right|^{\theta_{n}}\right) \leqq K^{n}\|t-s\|^{n+n \delta}$ for all $t, s$, in $H$, then almost all sample functions of the process are continuous in compact subsets of $\left\{t: a_{n} \leqq t_{n} \leqq a_{n}+1 / 2^{n}\right\}$.

Proof. The proof is exactly that of Theorem 2 if we replace the estimate

$$
P\left\{x:\left|x\left(q_{i}\right)-x\left(p_{j}\right)\right|>a_{n}\right\} \leqq \frac{2}{\sqrt{2 \pi}\left(\frac{a_{n}}{\sigma_{n}}\right)} \exp \left(-\frac{1}{2}\left(\frac{a_{n}}{\sigma_{n}}\right)^{2}\right)
$$

valid for Gaussian processes, by the weaker generalized Chebyshev estimate

$$
P\left\{x:\left|x\left(q_{i}\right)-x\left(p_{j}\right)\right|>a_{n}\right\} \leqq \frac{E\left(\left|\xi_{t}-\xi_{s}\right| Q_{n}\right)}{a_{n}^{Q_{n}}} \leqq \frac{K^{n}\|t-s\|^{n+n \delta}}{a_{n}^{Q_{n}}}
$$

which is valid for general processes. 
THEOREM 4. Let $\left\{\xi_{t}: t \in T\right\}$ be a process with $T=\left\{\left(t_{1}, t_{2}, \cdots t_{Q}\right)\right.$ : $\left.0 \leqq t_{i} \leqq 1, i=1,2, \cdots Q\right\}$ and $E\left(\xi_{t}\right)=0$. If there are constants $\lambda>0, \alpha>0$ and $K$ such that

$$
E\left(\left|\xi_{t}-\xi_{s}\right|^{\lambda}\right) \leqq k\|t-s\|^{Q+\alpha}
$$

for all $t, s$ in $T$, then almost all sample functions of the process are Lipschitz- $\beta$ continuous for $0<\beta<\alpha / \lambda$.

Proof. The proof is the Q-dimensional analogue of Theorem 1 with the following modifications.

Choose $\theta$ such that $0<\beta<\theta<\alpha / \lambda$.

Observe that in the case here of general processes we may use the generalized Chebyshev inequality to obtain the following estimate:

$$
\begin{aligned}
& P\left\{x: \mid x\left(\left(\vec{k}+\varepsilon_{i}\right) h_{n}-x\left(\vec{k} h_{n}\right) \mid \geqq h_{n}^{\theta}\right\}\right. \\
& \quad \leqq \frac{E\left(\left|x\left(\left(\vec{k}+\varepsilon_{i}\right) h_{n}\right)-x\left(\vec{k} h_{n}\right)\right|^{\gamma}\right)}{\left(h_{n}^{\theta}\right)^{\lambda}} \leqq \frac{k h_{n}^{\theta+\alpha}}{h_{n}^{\theta \lambda}}=\frac{k}{2^{n(Q+\alpha-\theta \lambda)}} .
\end{aligned}
$$

Hence

$$
\begin{gathered}
\sum_{n} P\left\{\max _{\vec{k}, \vec{i},\left(\vec{k}+\varepsilon_{i}\right) h_{n} \in D, \vec{k} h_{n} \in D}\left|x\left(\left(\vec{k}+\varepsilon_{i}\right) h_{n}\right)-x\left(\vec{k} h_{n}\right)\right| \geqq \frac{1}{2^{n \theta}}\right\} \\
\leqq \\
\sum_{n}\left(2^{n}\right)^{\ell} Q\left(\frac{k}{2^{n(Q+\alpha-\theta \lambda)}}\right)=\sum_{n} \frac{Q k}{2^{n(\alpha-\theta \lambda)}}<\infty \text { since } \alpha-\theta \lambda>0 .
\end{gathered}
$$

Now apply the Borel-Cantelli lemma to conclude that for almost all sample functions $x$, there is an integer $N$ (dependent on $x$ ) such that $n \geqq N$ implies that

$$
\left|x\left(\left(\vec{k}+\varepsilon_{i}\right) h_{n}\right)-x\left(\vec{k} h_{n}\right)\right|<\frac{1}{2^{n \theta}}
$$

for all $\vec{k}, i$ such that $\left(\vec{k}-\varepsilon_{i}\right) h_{n}$ and $\vec{k} h_{n}$ are in $D$.

Observe that inequality (6) here is identical to inequality (2) in the proof of Theorem 1. From this point onward, the two proofs are identical except $\alpha / 2$ should be replaced by $\alpha / \lambda$, and $\infty$-dimensional computations replaced by $Q$-dimensional computations.

4. Levy's Brownian process. Paul Levy defined a Gaussian process $\left\{\xi_{t} ; t \in H\right\}$ where the parameter space $H$ is the Hilbert space $l_{2}$ by specifying that

(1) $E\left(\xi_{t}\right)=0$ for all $t \in H$

(2) $E\left(\xi_{t} \xi_{s}\right)=(1 / 2)\{\|t\|+\|s\|-\|t-s\|\}$.

He showed that this process has the property that almost all sample functions are discontinuous in the Hilbert sphere. [3] 
In the following we shall describe the process further by showing that in every compact subset $T$ of $\left\{t: a_{n} \leqq t_{n} \leqq a_{n}+1 / 2^{n}\right\}$ almost all sample functions are Lipschitz- $\beta$ continuous for $0<\beta<1 / 2$ whereas in every noncompact subset $T$ of $H$ of the form $T=\left\{\left(t_{1}, t_{2}, \cdots t_{n}, \cdots\right)\right.$ : $\left.a_{n} \leqq t_{n} \leqq b_{n}\right\}$, almost all sample functions are discontinuous.

Lemma 3 [6]. Let $T=\left\{t=\left(t_{1}, t_{2}, \cdots t_{n}, \cdots\right): 0 \leqq t_{n} \leqq a_{n}\right\}, a_{n} \neq \infty$. $T$ is compact if and only if $\sum_{n=1}^{\infty} a_{n}^{2}<\infty$.

In the next lemma, $\left\{A_{n}\right\}$ is any sequence in $l_{2}$ with the property that $\left\|A_{n}\right\|=1$ and $\left\|A_{i}-A_{j}\right\|=\sqrt{2}, i \neq j$.

LEMma 4. Let $X$ be the Brownian process defined by the covariance function $r(s, t)=1 / 2\{\|s\|+\|t\|-\|s-t\|\}$ and $E\left(X_{t}\right)=0$. Set $X_{n}=X\left(A_{n}\right)$. If $X_{1}, X_{2}, \cdots X_{n}$ are known then

$$
X_{n+1}=\mu_{n}+\sigma_{n} \tilde{\xi}_{n}
$$

where

$$
\begin{aligned}
& \mu_{n}=\frac{1}{n}\left(X_{1}+X_{2} \cdots+X_{n}\right) \\
& \sigma_{n}^{2}=\frac{1}{\sqrt{2}}+\frac{1}{n \sqrt{2}} \\
& \tilde{\xi}_{n}=\text { Gaussian random variable with mean } 0 \text {, variance } 1 . \\
& \text { Proof. Using the formula of Paul Levy [5], we have }
\end{aligned}
$$

$$
X(B)-X(A)=\xi \sqrt{\|A-B\|}
$$

where $\xi$ is a Gaussian random variable with mean 0 and variance 1. Hence we have

$$
\left\{\begin{array}{c}
X\left(A_{n+1}\right)-X\left(A_{1}\right)=X_{n+1}-X_{n}=\xi_{1} \sqrt{\sqrt{\overline{2}}} \\
\vdots \\
X\left(A_{n+1}\right)-X\left(A_{n}\right)=X_{n+1}-X_{n}=\xi_{n} \sqrt{\sqrt{\overline{2}}}
\end{array}\right.
$$

where $\xi_{1}, \xi_{2}, \cdots \xi_{n}$ are Gaussian random variables with mean 0 and variance 1 .

Summing equations (7) and dividing by $n$, we have

$$
\begin{aligned}
X_{n+1} & =\frac{X_{1}+X_{2}+\cdots+X_{n}}{n}+\frac{\sqrt{\sqrt{2}}}{n}\left\{\xi_{1}+\xi_{2} \cdots+\xi_{n}\right\} \\
& =\mu_{n}+\sigma_{n} \tilde{\xi}_{n}
\end{aligned}
$$

where 


$$
\mu_{n}=\frac{1}{n}\left(X_{1}+X_{2} \cdots+X_{n}\right)
$$

and

$$
\begin{aligned}
\sigma_{n}^{2} & =E\left\{\left[\frac{\sqrt{\sqrt{2}}}{n}\left(\xi_{1}+\xi_{2}+\cdots+\xi_{n}\right)\right]^{2}\right\} \\
& =\frac{\sqrt{2}}{n^{2}} E\left\{\left(\xi_{1}+\xi_{2}+\cdots+\xi_{n}\right)^{2}\right\} \\
& =\frac{\sqrt{2}}{n^{2}}(n) E\left\{\xi_{1}^{2}\right\}+\frac{\sqrt{2}}{n^{2}} n(n-1) E\left\{\xi_{1} \xi_{2}\right\}
\end{aligned}
$$

when we use the fact that $E\left(\xi_{1}^{2}\right)=E\left(\xi_{j}^{2}\right)$ for all $j=1, \cdots n$; and $E\left(\xi_{1} \xi_{2}\right)=E\left(\xi_{i} \xi_{j}\right)$ for all $i \neq j . \quad E\left(\xi_{1}^{2}\right)=1$;

$E\left(\xi_{1} \xi_{2}\right)$

$$
\begin{aligned}
& =E\left\{\frac{1}{\sqrt{\sqrt{2}}}\left(X_{n+1}-X_{1}\right) \frac{1}{\sqrt{\sqrt{2}}}\left(X_{n+1}-X_{2}\right)\right\} \\
& =\frac{1}{\sqrt{2}} E\left\{X_{n+1}^{2}-X_{1} X_{n+1}-X_{2} X_{n+1}+X_{1} X_{2}\right\} \\
& =\frac{1}{\sqrt{2}}\left\{1-\frac{1}{2}(1+1-\sqrt{2})-\frac{1}{2}(1+1-\sqrt{2})+\frac{1}{2}(1+1-\sqrt{2})\right\} \\
& =\frac{1}{\sqrt{2}}\left\{1-\frac{1}{2}(2-\sqrt{2})\right\}=\frac{1}{\sqrt{2}}\left\{1-1+\frac{\sqrt{2}}{2}\right\}=\frac{1}{2} .
\end{aligned}
$$

Hence

$$
\begin{aligned}
\sigma_{n}^{2} & =\left(\frac{\sqrt{2}}{n^{2}}\right) n+\left(\frac{\sqrt{2}}{n^{2}}\right) n(n-1) \frac{1}{2}=\frac{\sqrt{2}}{n}+\frac{\sqrt{2}}{2 n}(n-1) \\
& =\frac{\sqrt{2}}{n}+\frac{\sqrt{2}}{2}-\frac{\sqrt{2}}{2 n}=\frac{1}{\sqrt{2}}+\frac{1}{n \sqrt{2}} .
\end{aligned}
$$

THEOREM 5. Let $\left\{\xi_{t} ; t \in H\right\}$ be the Brownian process defined by the covariance function $r(s, t)=1 / 2\{\|s\|+\|t\|-\|s-t\|\}$ and $E\left(\xi_{t}\right)=0$. Let $T$ be a subset of $H$.

(1) Almost all sample functions of the process are Lipschitz- $\beta$ continuous, $0<\beta<(1 / 2)$, in $T$ if $T$ is a compact subset of a set of the form $\left\{\left(t_{1}, t_{2}, \cdots t_{n}, \cdots\right): a_{n} \leqq t_{n} \leqq a_{n}+\left(1 / 2^{n}\right)\right\}$.

(2) Almost all sample functions of the process are discontinuous in $T$ if $T$ is not compact, and of the form $T=\left\{\left(t_{1}, t_{2}, \cdots t_{n}, \cdots\right)\right.$ : $\left.a_{n} \leqq t_{n} \leqq b_{n}\right\}$.

Proof. Part (1) follows from Theorem 1 since 


$$
\begin{aligned}
E\left(\left|\xi_{t}-\xi_{s}\right|^{2}\right) & =r(t, t)-2 r(t, s)-r(s, s) \\
& =\|t\|-\{\|s\|+\|t\|-\|s-t\|\}+\|s\| \\
& =\|t-s\|
\end{aligned}
$$

so that conditions of the theorem are satisfied for $\alpha=1$. Hence we need only prove part (2).

Without loss of generality, we may assume that $T$ is of the form $T=\left\{t=\left(t_{1}, \cdots t_{n}, \cdots\right): 0 \leqq t_{n} \leqq a_{n}\right\}$. By Lemma 3, we have $\sum a_{n}^{2}=\infty$ and hence there is a sequence $\left\{A_{n}\right\}$ in $T$ such that $\left\|A_{n}\right\|=1$, $\left\|A_{i}-A_{j}\right\|=\sqrt{2}$. Using the notation of Lemma 4 , we have

$$
X_{n+1}=\mu_{n}+\sigma_{n} \tilde{\xi}_{n}
$$

where

$$
\begin{aligned}
& \mu_{n}=\frac{1}{n}\left(X_{1}+X_{2} \cdots+X_{n}\right) \\
& \sigma_{n}^{2}=\frac{1}{\sqrt{2}}+\frac{1}{n \sqrt{2}} \\
& \widetilde{\xi}_{n}=\text { Gaussian random variable with mean } 0 \text { and variance } 1 .
\end{aligned}
$$

Let $M$ be an arbitrarily large real number. If we are given $\left|X_{1}\right|<$ $M,\left|X_{2}\right|<M, \cdots\left|X_{n}\right|<M$, then

$$
\mu_{n}=\frac{1}{n}\left(X_{1}+X_{2} \cdots+X_{n}\right)<M
$$

so that the conditional probability

$$
\begin{aligned}
& P\left\{\left|X_{n+1}\right|<M /\left|X_{1}\right|<M,\left|X_{2}\right|<M, \cdots\left|X_{n}\right|<M\right\} \\
& \quad=P\left\{\left|\mu_{n}+\sigma_{n} \widetilde{\xi}_{n}\right|<M\right\} \\
& \quad \leqq P\left\{\left|\sigma_{n} \tilde{\xi}_{n}\right|<M+\left|\mu_{n}\right|\right\} \leqq P\left\{\left|\sigma_{n} \tilde{\xi}_{n}\right|<2 M\right\} \\
& \quad \leqq P\left\{\left|\tilde{\xi}_{n}\right|<\frac{2 M}{\sigma_{n}}\right\} \leqq P\left\{\left|\tilde{\xi}_{n}\right|<\frac{2 M}{1 / \sqrt{\sqrt{2}}}\right\} \\
& \quad=K_{M}<1
\end{aligned}
$$

since

$$
\sigma_{n}<\frac{1}{\sqrt{\sqrt{2}}}
$$

Hence

$$
\begin{aligned}
& P\left\{\left|X_{1}\right|<M,\left|X_{2}\right|<M, \cdots\left|X_{n}\right|<M, \cdots\right\} \\
&= P\left\{\left|X_{1}\right|<M\right\} \cdot P\left\{\left|X_{2}\right|<M /\left|X_{1}\right|<M\right\} \cdots \\
& \times P\left\{\left|X_{n+1}\right|<M /\left|X_{1}\right|<M, \cdots\left|X_{n}\right|<M\right\} \cdots
\end{aligned}
$$




$$
\leqq \lim _{n \rightarrow \infty}\left(k_{M}\right)^{n}=0 \text { for any } M \text {. }
$$

Hence, the sequence $\left\{X_{n}\right\}$ is almost surely not bounded, which is to say that almost all sample functions of the process are discontinuous in $T$.

The proof of part 2 of Theorem 5 incorporates many of the steps in Paul Levy's proof that almost all sample functions of the Brownian process are discontinuous in the unit sphere.

\section{REFERENCES}

1. K. Borsuk, Analytic geometry in $n$ dimensions, (in Polish) (in particular Chapter IV.)

2. Z. Ciesielski, Hölder conditions for realizations of Gaussian processes, Trans. Amer. Math. Soc. 99, (1961).

3. Paul Levy, Le mouvement Brownien, Gauthier-Villars, Paris, 1954, 72.

4. - Processus stochastique et mouvement Brownien, Gauthier-Villars, Paris, 1948, 276-293.

5. - Random functions: A Laplacian random function depending on a point of Hilbert space, University of California Publications in Statistics, Vol. 2, No. 10, (1956), 195-206.

6. L. A. Lusternik and W. I. Sobolev, Introduction to functional analysis, Moskva, 1951 (in Russian) (in particular pp. 225-227).

7. V.S. Varadarajan, Notes from special topics in probability; Courant Institute of Mathematical Sciences, Spring, 1962 (especially p. 91, Proposition 11.3, Kolmogorov's theorem).

Received July 19, 1965. The material in this paper was included in the author's Doctoral Dissertation submitted to the Courant Institute of Mathematical Sciences of New York University, under the guidance of Professor M. Donsker. The research was supported in part by the Office of Naval Research under Contract NO. Nonr 285 (38).

Queens College of the City University of New York 



\section{PACIFIC JOURNAL OF MATHEMATICS}

\section{EDITORS}

\author{
H. SAMELSON \\ Stanford University \\ Stanford, California \\ J. P. JANS \\ University of Washington \\ Seattle, Washington 98105
}

\author{
J. DUGUNDJI \\ University of Southern California \\ Los Angeles, California 90007 \\ RICHARD ARENS \\ University of California \\ Los Angeles, California 90024
}

\section{ASSOCIATE EDITORS}
E. F. BECKENBACH
B. H. NeumanN
F. WOLF
K. YOSIDA

\section{SUPPORTING INSTITUTIONS}

\author{
UNIVERSITY OF BRITISH COLUMBIA \\ CALIFORNIA INSTITUTE OF TECHNOLOGY \\ UNIVERSITY OF CALIFORNIA \\ MONTANA STATE UNIVERSITY \\ UNIVERSITY OF NEVADA \\ NEW MEXICO STATE UNIVERSITY \\ OREGON STATE UNIVERSITY \\ UNIVERSITY OF OREGON \\ OSAKA UNIVERSITY \\ UNIVERSITY OF SOUTHERN CALIFORNIA
}

STANFORD UNIVERSITY

UNIVERSITY OF TOKYO

UNIVERSITY OF UTAH

WASHINGTON STATE UNIVERSITY

UNIVERSITY OF WASHINGTON

AMERICAN MATHEMATICAL SOCIETY CHEVRON RESEARCH CORPORATION TRW SYSTEMS

NAVAL ORDNANCE TEST STATION 


\section{Pacific Journal of Mathematics}

\section{Vol. 19, No. 1 \\ May, 1966}

A. R. Brodsky, The existence of wave operators for nonlinear equations... 1

Gulbank D. Chakerian, Sets of constant width................... 13

Robert Ray Colby, On indecomposable modules over rings with minimum condition....................................... 23

James Robert Dorroh, Contraction semi-groups in a function space ....... 35

Victor A. Dulock and Harold V. McIntosh, On the degeneracy of the Kepler

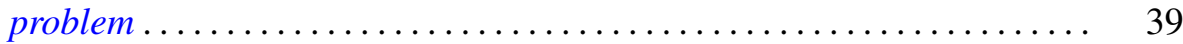

James Arthur Dyer, The inversion of a class of linear operators ......... 57

N. S. Gopalakrishnan and Ramaiyengar Sridharan, Homological dimension of Ore-extensions ................................. 67

Daniel E. Gorenstein, On a theorem of Philip Hall ................. 77

Stanley P. Gudder, Uniqueness and existence properties of bounded observables..................................... 81

Ronald Joseph Miech, An asymptotic property of the Euler function ....... 95

Peter Alexander Rejto, On the essential spectrum of the hydrogen energy and related operators ............................... 109

Duane Sather, Maximum and monotonicity properties of initial boundary

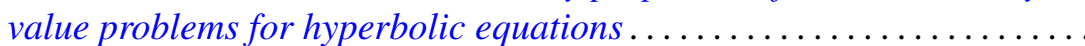

Peggy Strait, Sample function regularity for Gaussian processes with the parameter in a Hilbert space ........................... 159

Donald Reginald Traylor, Metrizability in normal Moore spaces ........... 175

Uppuluri V. Ramamohana Rao, On a stronger version of Wallis' formula ...............................

Adil Mohamed Yaqub, Some classes of ring-logics....... 